12

\title{
Применение формулы Донкина в теории электростатических призм
}

\author{
(C) Ю.К. Голиков, ${ }^{1,2}$ А.С. Бердников, ${ }^{2,}$ Ф А.С. Антонов, ${ }^{2}$ Н.К. Краснова, ${ }^{1}$ К.В. Соловьёв ${ }^{1,2}$
}

${ }^{1}$ Санкт-Петербургский политехнический университет Петра Великого, 195251 Санкт-Петербург, Россия

${ }^{2}$ Институт аналитического приборостроения РАН, 190103 Санкт-Петербург, Россия

ฯ e-mail: asberd@yandex.ru

(Поступило в Редакцию 22 сентября 2017 г. В окончательной редакции 24 февраля 2018 г.)

\begin{abstract}
Электростатическими призмами называются электронно- и ионно-оптические устройства, преобразующие параллельный пучок заряженных частиц на входе в параллельный же пучок на выходе, отклоняя пучок траекторий на угол, зависящий от энергии пучка. Принцип подобия траекторий для электрических полей, однородных по Эйлеру, обеспечивает для электростатических призм идеальные оптические свойства при применении полей с нулевым порядком однородности. Показано, что формула Донкина для трехмерных однородных гармонических функций обеспечивает доступ к максимально широкому классу аналитических выражений, задающих однородные электрические потенциалы нулевого порядка. Рассмотрены примеры электростатических призм, рассчитанных с помощью формулы Донкина.
\end{abstract}

DOI: $10.21883 /$ JTF.2018.11.46635.2498

\section{Введение}

Настоящая работа продолжает исследования электронно-оптических свойств электрических полей, положительно однородных по Эйлеру с нулевым порядком однородности, которые были начаты в публикациях $[1,2]$. Электростатическое поле является однородным по Эйлеру с порядком однородности, равным $n$, если напряженность электрического поля $\mathbf{E}(x, y, z)$ как функция пространственных координат удовлетворяет тождеству

$$
\mathbf{E}(\lambda x, \lambda y, \lambda z) \equiv \lambda^{n-1} \mathbf{E}(x, y, z)
$$

в области, в которой происходит движение заряженных частиц, при всех $\lambda>0$. Простейшими примерами полей, однородных по Эйлеру, являются поле электростатической квадрупольной линзы с потенциалом $U_{0}\left(x^{2}-y^{2}\right)[3]$ и поле точечного заряда с потенциалом $U_{0} / \sqrt{x^{2}+y^{2}+z^{2}}$.

Свойство (1) будет выполнено, если потенциал электрического поля $U(x, y, z)$ будет функцией, положительно однородной по Эйлеру с порядком однородности $n$ в смысле, который придается этому термину в общих курсах математического анализа $[4,5]$ : $\forall \lambda>0: U(\lambda x, \lambda y, \lambda z)=\equiv \lambda^{n} U(x, y, z)$. Исключительные случаи, когда электрический потенциал не является функцией, однородной по Эйлеру, но при этом порождаемое им электрическое поле тем не менее будет однородным по Эйлеру, исследуются в [6,7]. Показано, что все такие случаи сводятся к выражениям вида

$$
U(x, y, z)=V(x, y, z)+U_{0} \log \left(z+\sqrt{x^{2}+y^{2}+z^{2}}\right),
$$

где $V(x, y, z)$ будет однородной гармонической функцией нулевого порядка и определяется по формуле Донкина (4) (см. далее), а $U_{0}$ - произвольная константа [6,7].
Принцип подобия траекторий [8] постулирует, что если для траектории заряженной частицы в электрическом поле, однородном по Эйлеру, пропорционально изменить начальные условия, - а именно, масштабировать в $\lambda$ раз начальные координаты, сохранить начальные углы вылета и масштабировать в $\lambda^{n}$ раз начальную кинетическую энергию, - то ее траектория будет представлять собой геометрически масштабированную в $\lambda$ раз исходную траекторию с временем движения, масштабированным в $\lambda^{(n-2) 2}$ раз. Из принципа подобия траекторий следует, что если порядок однородности электрического поля $n$ равен нулю, то при масштабировании начальных координат и сохранении неизменными начальной кинетической энергии и начальных углов будет происходить пропорциональное масштабирование траекторий заряженных частиц.

В итоге в электрических полях, однородных по Эйлеру с нулевым порядком однородности, заряженные частицы, стартующие параллельно друг другу с масштабированными начальными координатами и фиксированной начальной энергией, будут обязаны двигаться по масштабированным траекториям. В свою очередь это означает, что если на входе такого электрического поля имеется параллельный моноэнергетический пучок заряженных частиц с разбросом начальных координат, который двигается в плоскости симметрии поля, то на выходе из электрического поля также получится параллельный пучок заряженных частиц. Угол поворота пучка и поперечная компрессия пучка будут зависеть от начальной кинетической энергии и угла влета, но при этом входные параллельные моноэнергетические траектории будут оставаться строго параллельными на выходе. В качестве примера на рис. 1, $a$ показаны траектории моноэнергетических параллельных пучков для поворотной системы [9], имеющей вид электростатиче- 


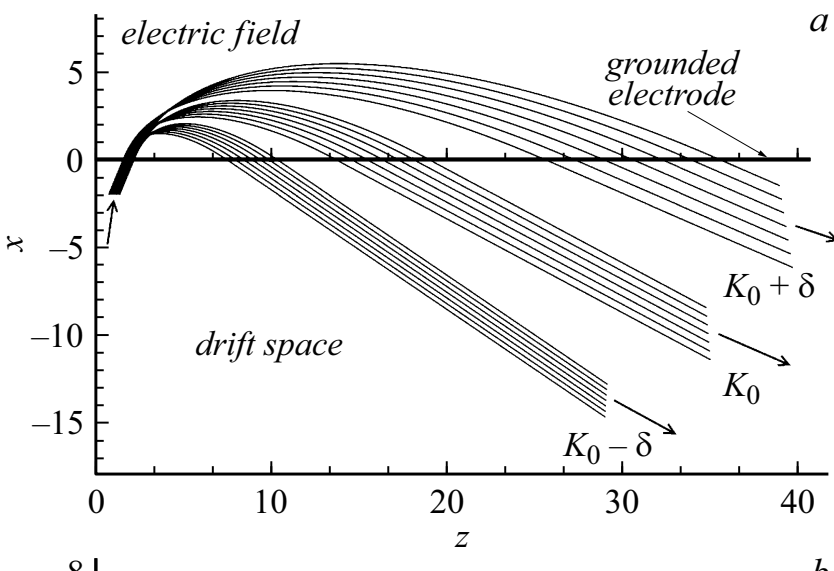

$b$

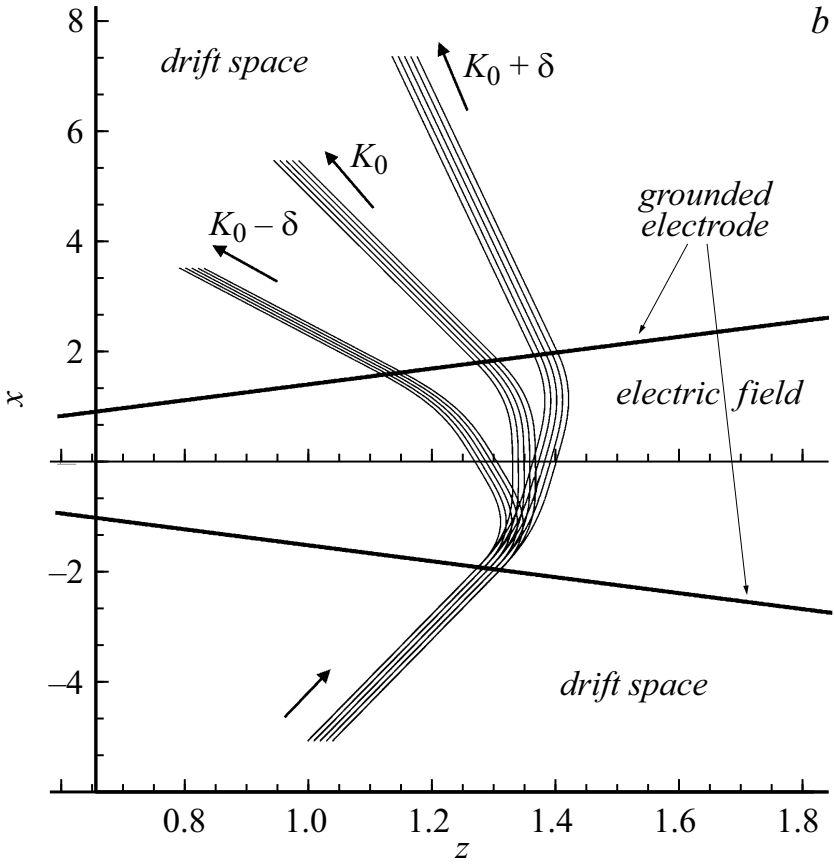

Рис. 1. Отклонение параллельного пучка моноэнергетических траекторий с кинетическими энергиями $K_{0}-\delta, K_{0}$ и $K_{0}+\delta$ соответственно в электрическом поле, однородном по Эйлеру с нулевым порядком однородности: $a-$ поворотная система в виде электростатического зеркала с потенциалом (3), $b-$ электростатическая призма с потенциалом (6).

ского зеркала с потенциалом

$V(x, y, z)=\left\{\begin{array}{l}U_{0} \operatorname{arctg}\left(\frac{x}{a z-\left(\frac{1-a^{2}}{2 a}\right) \frac{x^{2}+y^{2}}{z+\sqrt{x^{2}+y^{2}+z^{2}}}}\right) \text { при } x \geq 0, \\ 0 \text { при } x \leq 0\end{array}\right.$

(здесь параметр $U_{0}$ характеризует разность потенциалов между электродами, а безразмерный параметр $a$, принадлежащий промежутку $0<a<1$, управляет геометрической формой электродов). Другим примером служат показанные на рис. $1, b$ траектории моноэнергетических параллельных пучков для электрического потенциала (6), преломляющего входной пучок частиц (см. далее разд. 2).
Электростатические устройства, преобразующие параллельные пучки на входе в параллельные пучки на выходе (телескопические системы с оптической точки зрения), называются электростатическими призмами, если происходит преломление пучка с сохранением в целом прежнего направления движения. Они играют важную роль в электронной и ионной оптике [10-12]. При разработке подобных устройств удобным инструментом оказываются электрические поля, однородные по Эйлеру с нулевым порядком однородности. В работах $[1,2]$ уже исследовались оптические свойства эйлеровских электрических потенциалов нулевого порядка, но лишь для поворотных устройств, когда выходной пучок двигается практически в обратном направлении (под тупым углом) по отношению к исходному пучку. В настоящей работе внимание сосредоточено на призматических устройствах, в которых направление движения выходного пучка не слишком существенно отклоняется от входного.

\section{1. Формула Донкина для однородных гармонических функций нулевого порядка}

Нахождение однородных по Эйлеру электрических потенциалов является непростой задачей, поскольку соответствующие функции $U(x, y, z)$ должны одновременно удовлетворять и уравнению Лапласа $U_{x x}+U_{y y}+U_{z z}=0$ и дифференциальному соотношению Эйлера для однородных функций $[4,5] x U_{x}+y U_{y}+z U_{z}=n U$. Для целочисленных порядков однородности имеется полное и законченное решение, позволяющее выражать трехмерные однородные гармонические функции заданного порядка через произвольные двумерные гармонические функции [13-16]. Имеется гарантия, что никакая однородная гармоническая функция не будет пропущена при последовательном применении указанного процесса (правда, при этом одна и та же трехмерная однородная гармоническая функция может получаться из разных двумерных гармонических функций). Для нецелочисленных порядков однородности задача далека от решения, хотя имеются полезные аналитические формулы, описывающие частные случаи трехмерных однородных гармонических функций с нецелочисленными порядками однородности [6].

Электрические потенциалы $V(x, y, z)$ с нулевым порядком однородности могут быть записаны с помощью формулы Донкина [1,2,13-15,17]:

$V(x, y, z)=F\left(\frac{x}{z+\sqrt{x^{2}+y^{2}+z^{2}}}, \frac{y}{z+\sqrt{x^{2}+y^{2}+z^{2}}}\right)$,

где $F(p, q)$ - произвольная функция, удовлетворяющая двумерному уравнению Лапласа $\partial^{2} F / \partial p^{2}+$ $+\partial^{2} F / \partial q^{2}=0$. При подстановке в формулу (4) произвольной гармонической функции $F(p, q)$ получается гар- 
моническая однородная функция $V(x, y, z)$ нулевого порядка. Справедливо и обратное утверждение: для любой гармонической однородной функции $V(x, y, z)$ нулевого порядка найдется подходящая гармоническая функция $F(p, q)$, с помощью которой функция $V(x, y, z)$ может быть представлена в виде (4). Разным функциям $F(p, q)$ соответствуют разные функции $V(x, y, z)$ и наоборот.

Формула Донкина (4) не является единственным выражением такого рода. Однако можно показать, что все другие алгебраические формулы вида (4), с помощью которых из произвольных двумерных гармонических функций получаются трехмерные гармонические функции с нулевым порядком однородности, выводятся из формулы Донкина с помощью конформной замены аргументов функции $F(p, q)[16]$. Некоторые из таких модификаций могут оказаться для конкретных задач более удобными, чем оригинальная формула (4).

Имеются и другие полезные выражения для гармонических функций с нулевым порядком однородности [1719], но не все они годятся для полного перебора однородных гармонических потенциалов нулевого порядка. Например, требованию „универсальности“ не будет удовлетворять интегральная формула Уиттекера [17], имеющая вид

$$
V(x, y, z)=\operatorname{Re} \int_{0}^{\pi} \log (x \cos t-y \sin t+i z) f(t) d t,
$$

в которой на произвольную комплекснозначную функцию $f(t)$ наложено ограничение $\operatorname{Re} \int_{0}^{\pi} f(t) d t=0$, или ее эквивалентная форма [19]:

$$
V(x, y, z)=\operatorname{Re} \int_{0}^{\pi} \frac{x \sin t+y \cos t}{x \cos t-y \sin t+i z} g(t) d t,
$$

где на комплекснозначную функцию $g(t)$ уже не накладывается никаких дополнительных ограничений. То же самое утверждение справедливо и для интегральных формул $[18,19]$, а также для альтернативных вариантов интегральных формул Уиттекера [27,28].

\section{2. Тригонометрические аналитические потенциалы}

У поворотных систем с электростатическими зеркалами вида $[1,2]$ для пучка заряженных частиц входная граница электрического поля является одновременно и выходной границей. Но в случае электростатической призмы пучок заряженных частиц проходит ее насквозь, так что у призмы должны быть разные геометрические границы для входа и для выхода. Следовательно, если только призма не является иммерсионной, электростатический потенциал $V(x, y, z)$ обязан иметь две разные эквипотенциальные поверхности с одним и тем же потенциалом (предположительно, нулевым), через которые осуществляется ввод и вывод заряженных частиц. Внутри же призмы в промежутке между этими двумя эквипотенциальными поверхностями имеется существенно ненулевое электрическое поле, которое управляет движением заряженных частиц и придает ему желаемые свойства.

Как правило, электрическое поле электронно-оптического или ионно-оптического устройства обладает плоскостью симметрии, так что базовая траектория, стартующая в плоскости симметрии, продолжает свое движение, не выходя из нее (здесь также требуется устойчивость пучка траекторий при малых отклонениях от плоскости симметрии в вертикальном направлении [20]). Для того чтобы электрический потенциал вида (4) обладал плоскостью симметрии $y=0$, необходимо и достаточно, чтобы функция $F(p, q)$ была четной по аргументу $q$. Возьмем для подстановки в формулу (4) функцию, которая является вещественной частью функции комплексного переменного $f(p+i q)=\cos \omega(p+i q)$ :

$$
F(p, q)=U_{0} \cos (\omega p) \operatorname{ch}(\omega q),
$$

где $\omega>0-$ произвольная константа. Этой функции соответствует однородный по Эйлеру трехмерный электростатический потенциал нулевого порядка, имеющий вид:

$$
\begin{aligned}
V(x, y, z)= & U_{0} \cos \left(\frac{\omega x}{z+\sqrt{x^{2}+y^{2}+z^{2}}}\right) \\
& \times \operatorname{ch}\left(\frac{\omega y}{z+\sqrt{x^{2}+y^{2}+z^{2}}}\right) .
\end{aligned}
$$

Вертикальные линии $p=(\pi / 2 \pm \pi k) / \omega$ (где $k=0,1,2, \ldots) \quad$ являются линиями постоянного нулевого потенциала для функции (рис. 2,a). Однако чтобы этим эквипотенциальным линиям соответствовали реальные эквипотенциальные поверхности функции $V(x, y, z)$, определенной в соответствии с формулой (6), константа должна быть достаточно велика: $\omega>\pi / 2 \approx 1.57$. При увеличении параметра $\omega$ уменьшается угол между двумя соседними эквипотенциальными поверхностями с нулевым потенциалом, так что с помощью $\omega$ можно управлять углом поворота призмы. ${ }^{1}$ При достаточно большом увеличении параметра $\omega$ у функции $V(x, y, z)$ появляются дополнительные эквипотенциальные поверхности с нулевым потенциалом, которые также можно использовать как входные и выходные электроды при конструировании электростатической призмы. Однако при таком выборе приходится заодно увеличивать и число вспомогательных электродов внутри призмы (что не всегда является

\footnotetext{
1 Также углом поворота призмы и ее оптическими свойствами можно управлять, выбирая для входа и выхода эквипотенциальные поверхности с равным, но ненулевым потенциалом. При этом для иммерсионной призмы входные и выходные эквипотенциальные поверхности могут иметь разный потенциал. Эти интересные возможности здесь не будут анализироваться.
} 

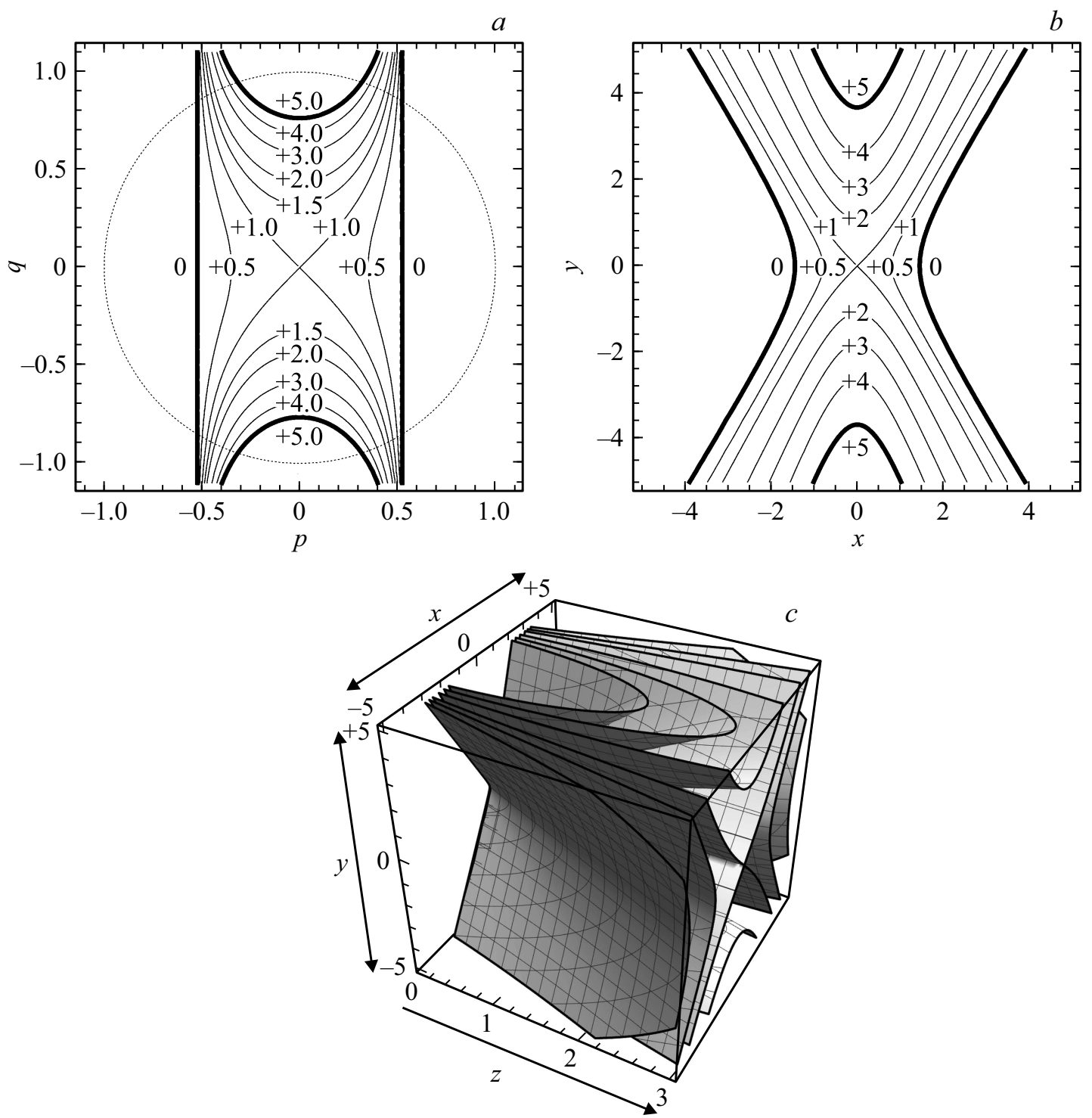

Pис. 2. Трехэлектродная симметричная электростатическая призма на базе электрического потенциала (6): $a-$ эквипотенциальные линии для функции (5) в координатах $(p, q)$ и окружность $p^{2}+q^{2}=1$, соответствующая бесконечно удаленным точкам трехмерного пространства, $b$ - сечения электродов и эквипотенциальные линии в трехмерном пространстве для плоскости $z=$ const, $c$ - трехмерная конфигурация электродов (эквипотенциальных поверхностей).

недостатком, поскольку увеличивает имеющиеся степени свободы при оптимизации характеристик устройства).

Для случая $\omega=3$ на рис. 2, $a$ показаны эквипотенциальные линии функции (5) на плоскости $(p, q)$. На рис. $2, b$ показано сечение электродов и эквипотенциальные линии в плоскости $z=1$ для трехмерного потенциала (6) (область пространства, лежащая вне электродов, выделенных жирными линиями, предполагается бесполевой). Квазиконические эквипотенциальные поверхности функции (6), которые можно использовать в качестве электродов, показаны на рис. 2, $c$.

На рис. $1, b$ для электрического потенциала (6) показаны лежащие в плоскости симметрии электростатического поля траектории монохроматических пучков положительно заряженных частиц, стартующих ортогонально к входному электроду с разными начальными кинетическими энергиями. Начальная кинетическая энергия центрального пучка и входной угол подбираются так, чтобы входная и выходная траектории были симметричны. Наглядно видно, что параллельные траектории на входе преобразуются в параллельные траектории на выходе, причем угол отклонения траекторий зависит от начальной кинетической энергии. Для проверки устойчивости траекторий при малых отклонении от центральной плоскости в вертикальном направлении достаточно воспользоваться критерием устойчивости, рассматриваемом в работе [20].

Функция (6) не единственный потенциал, подходящий для электростатических призм, идеальным образом преобразующих входные параллельные монохроматические 

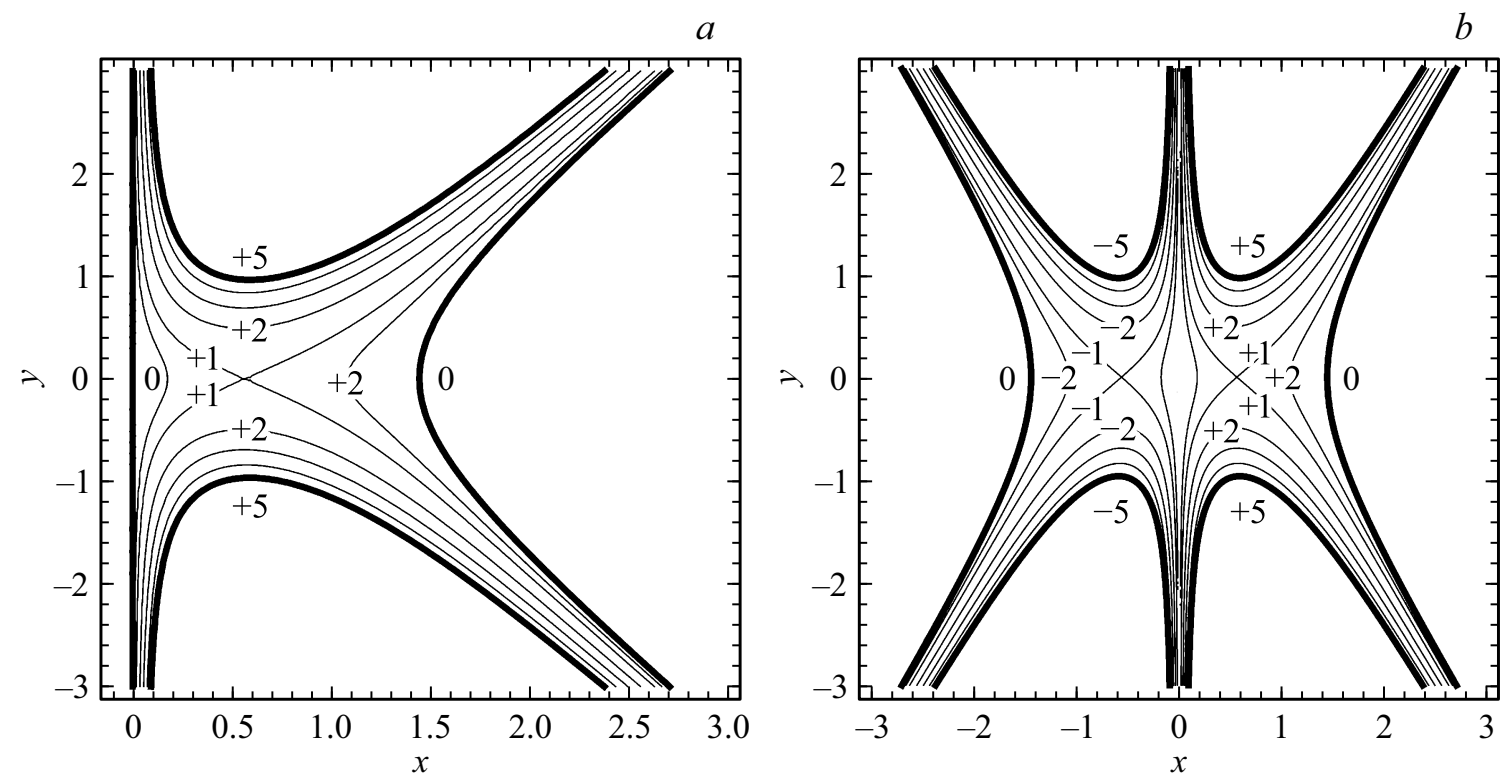

Pис. 3. Сечения электродов в плоскости $z=$ const для электростатической призмы на базе электрического потенциала (8): $a-$ несимметричная конфигурация электродов с входным электродом в виде заземленной вертикальной плоскости, $b-$ симметричная конфигурация криволинейных электродов с антисимметричными потенциалами.

пучки в выходные параллельные монохроматические пучки. Возьмем для формулы Донкина (4) функцию, которая является вещественной частью функции комплексного переменного $f(p+i q)=\sin \omega(p+i q)$ :

$$
F(p, q)=U_{0} \sin (\omega p) \operatorname{ch}(\omega q),
$$

где $\omega>0-$ произвольная константа. Чтобы у трехмерной гармонической функции

$$
\begin{aligned}
V(x, y, z)= & U_{0} \sin \left(\frac{\omega x}{z+\sqrt{x^{2}+y^{2}+z^{2}}}\right) \\
& \times \operatorname{ch}\left(\frac{\omega y}{z+\sqrt{x^{2}+y^{2}+z^{2}}}\right),
\end{aligned}
$$

которая получается при подстановке функции (7) в формулу Донкина (4), существовали эквипотенциальные поверхности с нулевым значением потенциала, которые можно использовать для входа и выхода в призму, константа $\omega$ должна удовлетворять условию $\omega>\pi \approx 3.14$. Эквипотенциальная линия $p=0$ с нулевым потенциалом для функции (7) отображается на эквипотенциальную поверхность $x=0$ для функции $V(x, y, z)$. Поэтому здесь входной (или выходной) электрод для трехэлектродной призмы может быть выбран как вертикальная плоскость, но при таком выборе призма перестает быть симметричной (рис. $3, a$ ). Симметрия возвращается, если эквипотенциальная поверхность $x=0$ перестает быть электродом и оказывается внутри призмы, а сама призма становится четырехэлектродной с антисимметриными напряжениями на внутренних электродах (рис. $3, b$ ).

Использование в выражениях для тригонометрических комплексных потенциалов сумм, состоящих из многих тригонометрических функций с общим периодом, приводит к еще более сложным электродным конфигурациям. В подобных системах, однако, появляются дополнительные степени свободы, позволяющие гибко управлять оптическими характеристиками электростатических призм. В случае, когда требуется иммерсионная электростатическая призма, синтез электродной конфигурации выполняется по аналогичной схеме, но для входного и для выходного электродов выбираются эквипотенциальные поверхности с разными потенциалами, соответствующими иммерсионному отношению. Подробное исследование этих возможностей выходит за рамки настоящей работы.

\section{3. Конструктивные электроды. Проблема краевых полей}

Достоинством однородных по Эйлеру потенциалов, рассмотренных в разд. 2, является то, что они представлены в простой аналитической форме, зависящей от нескольких свободных параметров. Это позволяет достаточно просто исследовать и оптимизировать их оптические свойства. Однако очень серьезным недостатком является то, что соответствующие этим потенциалам электроды имеют сложную геометрическую форму, не слишком конструктивную с точки зрения изготовлении реальных устройств. Например, среди рассмотренных в разд. 2 форм электродов только эквипотенциальная поверхность $x=0$ для донкиновского потенциала, порождаемого функцией (7), может рассматриваться как конструктивный электрод.

Другой проблемой является организация ввода и вывода заряженных частиц. В рассмотренных в разд. 2 

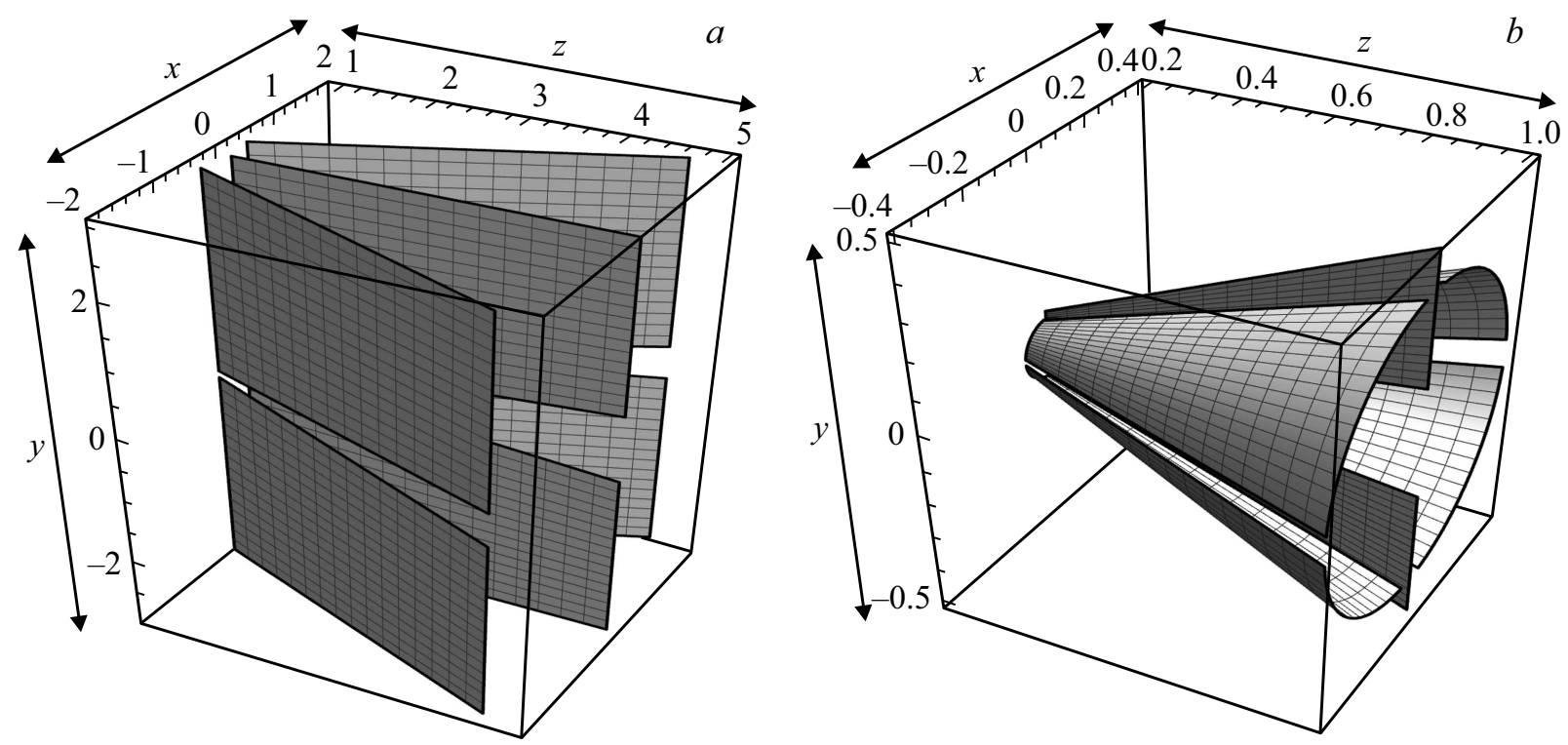

Рис. 4. Простейшие электростатические призмы с электродами конструктивной формы и бессеточными диафрагмами: $a-$ трехэлектродная призма с электродами в виде сегментов плоскостей с клиновидными щелями, $b$ - трехэлектродная призма с электродами в виде разрезанной центральной плоскости с фокусирующим потенциалом и заземленными сегментами круговых конусов.

примерах заряженные частицы вводились и выводились прямо сквозь бесконечно тонкий электрод, предполагая, что этот электрод представляет собой идеальную сетку. Реальная сетка, однако, серьезно ухудшит характеристики устройства. Гораздо лучше, если ввод и вывод осуществляются бессеточным образом через специально организованную диафрагму. Однако образующиеся при этом краевые поля не просто возмущают движение заряженных частиц. Скорее всего, полученное электрическое поле потеряет свое свойство быть однородным по Эйлеру. Это означает, что идеальные призматические свойства устройства также будут утеряны.

С данной проблемой позволяет справиться специальный подход к синтезу однородных по Эйлеру краевых полей, который рассматривается в [9,21]. Идея метода состоит в том, что исходный электрический потенциал для полупространства $z>0$ записывается в ,донкиновких “ъ координатах $(p, q, r)$ с помощью подстановки

$$
x=p r, \quad y=q r, \quad z=\frac{r}{2}\left(1-p^{2}-q^{2}\right),
$$

где обратное преобразование для области $p^{2}+q^{2}<1$, $r>0$ имеет вид

$$
\begin{gathered}
p=\frac{x}{z+\sqrt{x^{2}+y^{2}+z^{2}}}, \quad q=\frac{y}{z+\sqrt{x^{2}+y^{2}+z^{2}}}, \\
r=z+\sqrt{x^{2}+y^{2}+z^{2}} .
\end{gathered}
$$

Переменная $r$ в полученное в конечном итоге уравнение не входит из-за однородности трехмерного потенциала и поэтому выпадает из рассмотрения. Тогда краевая задача для трехмерного уравнения Лапласа с поверхностями-электродами и краевыми условиями Дирихле преобразуется в двумерную краевую задачу для двумерного уравнения Лапласа, где на плоскости $(p, q)$ границами с заданными краевыми условиями Дирихле будут криволинейные линии - образы электродов, полученные с помощью преобразования (9), (10).

Как и исходные электроды, эти новые границы являются сплошными. Однако если проделать в полученных двумерных границах дополнительные „дырки“ и решить соответствующую краевую задачу, то после обратного перехода от координат $(p, q, r)$ к координатам $(x, y, z)$ получим новый трехмерный электростатический потенциал, также однородный по Эйлеру с нулевым порядком однородности. Образы границ с „дырками“ превратятся в электроды с бессеточными диафрагмами, но для таких специальным образом синтезированных бессеточных диафрагм трехмерное электрическое поле сохранит свое свойство однородности по Эйлеру. Если „дырки“ достаточно малы по сравнению с типичными расстояниями между краевыми границами, то они не будут сильно искажать решение двумерного уравнения Лапласа. Поэтому для новой электродной конфигурации искажению подвергнуться лишь сами бессеточные диафрагмы и создаваемое ими краевое поле. Остальные же электроды, расположенные достаточно далеко от „дырок", сохранят свою прежнюю форму, точно также как и электрическое поле в основном объеме устройства вдали от привнесенных краевых полей практически останется прежним. Важно, что полученное электрическое поле будет однородным по Эйлеру во всех точках и, следовательно, сохранит свои идеальные призматические свойства. 


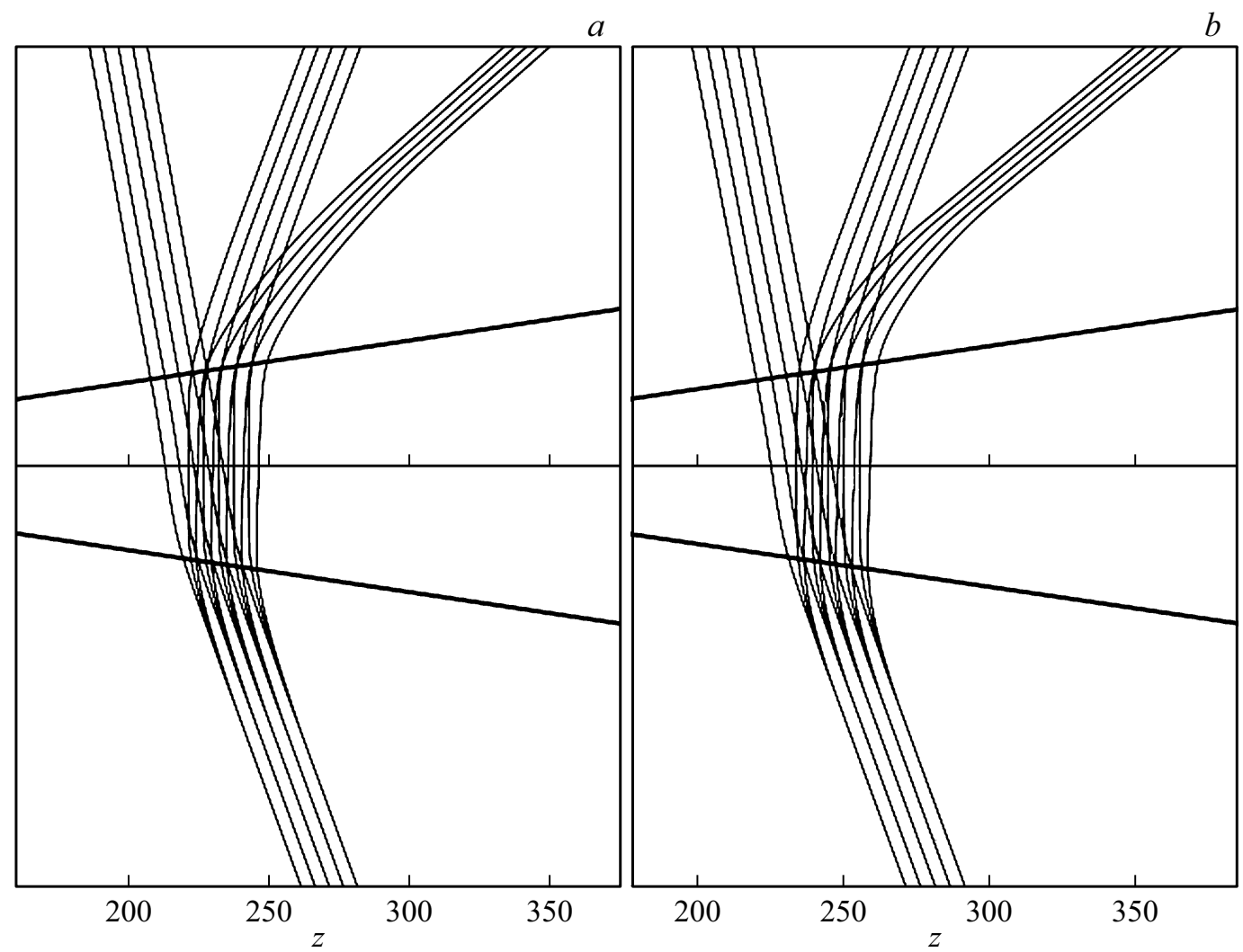

Рис. 5. Траектории параллельных монохроматических пучков заряженных частиц для электростатических призм, показанных на рис. 4: $a$ - для призмы с электродами в виде плоскостей с клиновидными щелями, $b$ - для призмы с электродами в виде центральной плоскости, окруженной коническими сегментами круговых конусов.

В частности, оказывается, что для электродов, соответствующих нулевому значению однородного по Эйлеру потенциала (такие электроды всегда имеют форму плоскости), синтезируемая бессеточная диафрагма для электрода-плоскости обязательно будет иметь вид клиновидной щели $[9,21]$. Однако при более внимательном рассмотрении оказывается, что для полей, однородных по Эйлеру с нулевым порядком однородности, любые „дырки“ при обратном переходе к трехмерному пространству превращаются в клиновидные щели соответствующего размера. Если добавленные клиновидные щели достаточно узкие (по сравнению с расстоянием между электродами), то для полученной электростатической призмы их наличие, конечно, несколько сместит угол поворота параллельного пучка и соответствующим образом изменит остальные оптические характеристики устройства, но при этом оставит в неприкосновенности базовое свойство призмы преобразовывать входные параллельные монохроматические пучки в выходные параллельные монохроматические пучки [9,21].

Специфика потенциалов, однородных по Эйлеру с нулевым порядком однородности, состоит в том, что тот же самый подход позволяет справиться и с проблемой неконструктивных электродов (для потенциалов, однородных по Эйлеру с ненулевыми порядками однородности, это не так). По-видимому, в качестве конструк- тивных электродов при создании электростатических призм допустимо ограничиться электродами, представляющими собой фрагменты плоскостей и круговых конусов, возможно, с клиновидными вырезами. Достаточно трудоемкий, но не представляющий особых проблем анализ показывает, что „отпечатками“ таких электродов на плоскости $(p, q)$ после замены переменных (9), (10) будут отрезки прямых и дуги окружностей (при этом отпечатками конических сегментов круговых конусов могут быть отрезки прямых, а отпечатками клиновидных сегментов плоскостей - дуги окружностей). Справедливо и обратное утверждение: кривые на плоскости $(p, q)$, являющиеся сегментами прямых и дугами окружностей, при обратном переходе к трехмерному пространству превращаются в конические сегменты круговых конусов и/или в клиновидные сегменты плоскостей.

В таком случае, выбрав на плоскости $(p, q)$ условные границы для электродов в виде отрезков прямых и дуг окружностей и решив соответствующую краевую задачу для двумерного уравнения Лапласа, мы не только получим после обратной замены переменных (10) трехмерное электрическое поле нулевого порядка, однородное по Эйлеру, но и гарантируем конструктивность трехмерных электродов, с помощью которых создается данное электрическое поле. С помощью общих методов теории функций комплексного переменного [22] 
и справочников по конформным отображениям при некоторой удаче для сконструированных таким образом двумерных краевых задач можно найти аналитическое решение. Можно также воспользоваться численными методами построения конформных отображений [23] или же решить соответствующую задачу Дирихле численно с помощью конечно-разностных методов или любого другого подходящего численного метода. Недостатком, однако, является тот факт, что кроме отдельных случаев специального вида у поставленной краевой задачи не будет решения в аналитической форме.

Численное или аналитическое решение для плоскости $(p, q)$ краевой задачи с границами в виде отрезков прямых и дуг окружностей порождает, в свою очередь, в пространстве $(x, y, z)$ трехмерный донкиновский потенциал $V(x, y, z)$ с конструктивными электродами. Это позволяет значительно увеличить возможности исследователя при конструировании с помощью формулы Донкина практически работающих электростатических призм с идеальными призматическими свойствами. В конечном счете, зачастую даже нет необходимости пользоваться формулой Донкина, поскольку заранее известно, что конфигурация электродов в виде конических сегментов круговых конусов и клиновидных сегментов плоскостей с клиновидными щелями порождает коническое электростатическое поле, однородное по Эйлеру с нулевым порядком однородности $[10,11]$, и тем самым обеспечивающее идеальные призматические свойства устройства. На рис. 4 показаны некоторые возможные конфигурации электродов для простейших электростатических призм, на рис. 5 - соответствующие им траектории, лежащие в плоскости симметрии и рассчитанные численно с помощью программы SIMION 8.01 [24]. Угол влета частиц равен $110^{\circ}$, энергия в единицах потенциала на центральном электроде равна $0.027,0.05,0.35$ (рис. $5, a)$ и $0.017,0.033,0.25$ (рис. $5, b$ ). Входная энергия центральной траектории подбиралась таким образом, чтобы обеспечить симметричность этой траектории. В силу этого траектории на рис. 5 оказываются похожими, хотя они и реализуются при разных кинетических энергиях заряженных частиц и в разных геометрических конфигурациях электродов. Также у электродных конфигураций на рис. 4, $a$ и рис. 4, $b$ будут разные фокусирующие свойства в вертикальном направлении и разные аберрационные характеристики.

\section{Заключение}

Аналитические потенциалы, являющиеся однородными по Эйлеру с нулевым порядком однородности, являются удобным средством для поиска электродных конфигураций для электростатических поворотных систем и для электростатических призм, обладающим свойством преобразовывать входные параллельные монохроматические пучки заряженных частиц (электронов, ионов) в выходные параллельные монохроматические пучки. Та- кие потенциалы позволяют быстро и эффективно исследовать оптические свойства систем с нетривиальными электродными конфигурациями и отбирать из них те электродные конфигурации, которые обладают перспективными (с точки зрения той или иной практической задачи) оптическими свойствами.

Представляется маловероятным, что подобные электродные конфигурации могут быть найдены методом „научного тыка“ или с помощью численной оптимизации произвольно взятой простой конфигурации электродов, если только исследователь не обладает априорной информацией, в каком именно классе систем надо искать оптимальное решение. Формула Донкина (4) или, в расширенном виде, формула Донкина с логарифмической добавкой (2) являются полезными инструментами при генерировании аналитических выражений для потенциалов однородных электрических полей нулевого порядка. Аналитические выражения, генерируемые формулами (2) и (4), позволяют быстро исследовать широкий массив возможных электрических потенциалов и отбирать из них те, которые обладают нужными исследователю оптическими характеристиками. В частности, при использовании для формул (2) и (4) тригонометрических функций комплексного переменного, обладающими в силу свойства периодичности входными и выходными заземленными электродами, возможно генерирование нетривиальных электродных конфигураций для электростатических призм.

Недостатком будет то, что получаемые на выходе криволинейные электроды сложной формы крайне неудобны при изготовлении и юстировке. Скорее всего, замена точных аналитических профилей электродов на огрубленные профили (клиновидные сегменты плоскостей и конические сегменты круговых конусов, описанные в разд. 3) в целом сохранит оптические свойства устройства, не слишком их испортив. Тогда численная оптимизация формы, расположения и потенциалов для огрубленных электродов позволит определить решение с приемлемыми оптическими характеристиками, оставаясь при этом в рамках конструктивных электродных конфигураций. Стоит подчеркнуть, что без информации о перспективном расположении электродов, которая получается из аналитических моделей, будет сложно искать подходящие конструктивные конфигурации, если только речь не идет о простых системах с простыми требованиями.

Авторы благодарны создателям, сотрудникам и спонсорам сайта rspl.royalsocietypublishing.org (Proceedings of the Royal Society of London), благодаря работе которых имеется возможность свободно знакомиться с уникальными и раритетными ссылками, в частности, с публикациями [13,14].

Для проведения и проверки аналитических выкладок, a также для изготовления рисунков использовалась программа Wolfram Mathematica версии 11 [25]. При редактировании рисунков использовалась свободно распространяемая программа Paint.NET версии 4 [26]. 
Настоящая работа выполнена в ИАП РАН в рамках гос. заказа № 007-00229-18-00.

\section{Список литературы}

[1] Габдуллин П.Г., Голиков Ю.К., Краснова Н.К., Давыдов С.Н. // ЖТФ. 2000. Т. 70. Вып. 2. С. 91-94.

[2] Габдуллин П.Г., Голиков Ю.К., Краснова Н.К., Давыдов С.Н. // ЖТФ. 2000. Т. 70. Вып. 3. С. 44-47.

[3] Явор С.Я. Фокусировка заряженных частиц квадрупольными линзами. М.: Атомиздат, 1968. 263 с.

[4] Фихтенгольи, Г.М. Курс дифференциального и интегрального исчисления. Т. 1. М.: Физматлит, 2001. 616 с.

[5] Смирнов В.И. Курс высшей математики. Т. 1. СПб: БХВПетербург, 2008. 616 с.

[6] Бердников А.С., Аверин И.А., Краснова Н.К., Соловьёв К.В. // Вестник Актюбинского регион. гос. ун-та им. К. Жубанова. Физико-математические науки. 2016. № 2 (54). C. 147-164.

[7] Бердников А.С., Аверин И.А., Краснова Н.К., Соловъёв К.В. // Успехи прикладной физики. 2017. Т. 5. № 1. C. $10-27$.

[8] Голиков Ю.К., Краснова Н.К. // Прикладная физика. 2007. № 2. C. 5-11.

[9] Голиков Ю.К., Бердников А.С., Антонов А.С., Краснова Н.К., Соловьёв К.В. // ЖТФ. 2018. Т. 88. Вып. 4. С. 609 613.

[10] Кельман В.М., Карецкая С.П., Федулина Л.В., Якушев E.M. Электронно-оптические элементы призменных спектрометров заряженных частиц. Алма-Ата: Наука, 1979.

[11] Кельман В.М., Родникова И.В., Секунова Л.М. Статические масс-спектрометры. Алма-Ата: Наука, 1985. 264 с.

[12] Лукашевич В.B. // Физика элементарных частиц и атомного ядра. 2003. Т. 34. Вып. 6. С. 1520-1562.

[13] Donkin W.F. // Philosophical Transactions of the Royal Society of London. 1857. Vol. 147. P. 43-57.

[14] Donkin W.F. // Proceedings of the Royal Society of London. 1856-1857. Vol. 8. P. 307-310.

[15] Гобсон Е.В. Теория сферических и эллипсоидальных функций. М.: ИЛ, 1952. 476 с.

[16] Бердников А.С., Аверин И.А., Краснова Н.К., Соловьёв К.В. // Научное приборостроение. 2016. Т. 26. № 4. C. 13-30.

[17] Уиттекер Э.Т., Ватсон Джс. Курс современного анализа. Ч. 2: Трансцендентные функции. М.: ГИФМЛ, 1963. 516 с.

[18] Голиков Ю.К. // Вестник Актюбинского регион. гос. унта им. К. Жубанова. Физико-математические науки. 2016. № 2 (54). C. 59-62.

[19] Голиков Ю.К. // Вестник Актюбинского регион. гос. унта им. К. Жубанова. Физико-математические науки. 2016. № 2 (44). C. 165-181.

[20] Бердников А.С., Краснова Н.К. // Научное приборостроение. 2015. Т. 25. № 2. С. 69-90.

[21] Аверин И.А., Бердников А.С. // Успехи прикладной физики. 2016. Т. 4. № 1. С. 5-8.

[22] Лаврентьев М.А., Шабат Б.В. Методы теории функций комплексного переменного. М.: Наука, 1965. 716 c.

[23] Фильчаков П.Ф. Приближенные методы конформных отображений. Киев: Наукова думка, 1964. 532 с.
[24] SIMION: Ion and Electron Optics Simulator // URL: $\mathrm{http} / / /$ simion.com

[25] Wolfram Mathematica: the system for modern technical computing // URL: http://wolfram.com/mathematica/

[26] Paint.NET: Free Software for Digital Photo Editing // URL: http://www.getpaint.net

[27] Бердников А.С., Краснова Н.К., Соловьев К.В. // Научное приборостроение. 2017. Т. 27. № 4. С. 63-71.

[27] Бердников А.С., Краснова Н.К., Соловьев К.В. // Научное приборостроение. 2017. Т. 27. № 4. С. 72-79. 